\title{
CFA BASED UNIVERSAL BIQUAD FILTER
}

\author{
Maria Cristina Piccirilli, \\ Department of Electronics and Telecommunications, University of Florence, Firenze, Italy \\ piccirilli@det.unifi.it
}

DOI: 10.36724/2664-066X-202I-7-3-2-5

\begin{abstract}
A universal biquad filter using current feedback amplifiers (CFA) as active devices and a canonical number of capacitors is presented. It realizes the classical feedforward structure through the use of grounded capacitors and MOS transistors, which permit a full integrability. The proposed circuit has attractive characteristics, as the possibility to electronically control the transfer function coefficients and the possibility to be directly inserted within cascaded configurations. The theoretical analysis of the circuit is followed by some PSPICE simulation results in agreement with the theoretical findings. In this paper a universal fully integrable biquad using CFAs as active devices and a canonical number of capacitors is presented. It realizes the classical feedforward structure [3] through the use of grounded capacitors and MOS transistors. The presence of grounded capacitors makes the proposed circuit absorb shunt parasitic capacitances. All the possible kinds of responses can be realized, while in the biquad reported in [2] only the low-pass and band-pass responses can be obtained. The numerator and denominator coefficients of the voltage transfer function of the proposed biquad can be electronically controlled by acting on the gate voltages of the MOS transistors of the circuit. Moreover the presented structure has a low-impedance output and then it can be cascaded without additional buffers.
\end{abstract}

KEYWORDS: Analog circuits, continuous-time filters. 


\section{Introduction}

Continuous-time filters realizable in fully integrated form continue to receive considerable attention. The techniques appeared in literature in the last years are generally based on the use of active devices alternative to the classical operational amplifier, as, for example, the operational transconductance amplifier (OTA) and the second generation current conveyor (CCII). Until now a relatively little attention has been given to the use of the current feedback amplifier (CFA) in the realization of fully integrated continuous-time filters. The CFA is an amplifier exploiting a circuit topology that emphasizes current mode operation, which is inherently much faster than voltage mode operation [1]. Differently from classical op amps, whose dynamics are limited by the gain-bandwidth product and slew rate, CFAs deliver high bandwidth and slew rate. An example of fully integrated continuous-time filter based on the use of the CFA is reported in [2], where a Tow- Thomas biquad containing capacitors and MOS transistors is presented.

The circuit has been simulated with PSPICE and the simulation results agree with the theoretical analysis.

\section{Circuit description}

A classical biquad with op amps, resistors and capacitors is the feedforward circuit [3]. The goal is to obtain a fully integrable version of this biquad by using CFAs instead of classical op amps and by replacing the resistors of the corresponding $\mathrm{RC}$ circuit with suitable configurations of MOS transistors as the MRC. The MRC (MOS Resistive Circuit), shown in Fig. 1, is a cell constituted by four matched MOS transistors operating in nonsaturation region, with gate voltages equal two by two, which allows to completely cancel the channel resistance nonlinearities of MOS transistors [4].

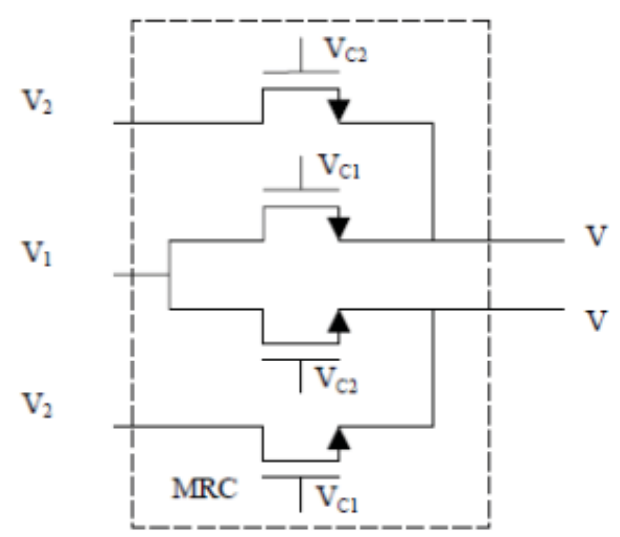

Fig. 1. MRC circuit

In Fig. 2 the fully integrable feedforward biquad is shown. The transfer function is obtained by proceeding in the following way. An ideal model for the CFA is considered, in which the current at the input node $\mathrm{Y}$ is zero, the current at the input node $\mathrm{X}$ is equal to the current at the internal node $\mathrm{Z}$, the voltages relevant to the nodes $\mathrm{Y}$ and
$\mathrm{X}$ are equal and a unity gain buffer is placed between the internal node $\mathrm{Z}$ and the CFA output, so making ideally null the output impedance (this feature permits the direct insertion of the proposed circuit in cascaded configurations). For what concerns the MRC, by supposing, without loss of generality, to use n-channel MOS transistors, the channel current ID in nonsaturation region can be written as follows:

$$
I_{D}=F\left(V_{D}, V c\right)-F\left(V_{S}, V_{C}\right)
$$

where $V_{D}, V_{S}$ and $V_{C}$ are the drain, source and gate voltages respectively. By exploiting the property that $F\left(V_{A}, V_{B}\right)$ $F\left(V_{A}, V_{C}\right)=2 K V_{A}\left(V_{B^{-}} V_{C}\right)$ [4], where $K$ is a constant depending on the implementation characteristics of the MOS transistors [4], a routine analysis as in [2] yields the following expression for the transfer function:

$$
\begin{aligned}
& \frac{V_{0}(s)}{V_{i}(s)}=\frac{\frac{K_{2}\left(V_{C 7}-V_{C S}\right)}{K_{8}\left(V_{C S}-V_{C 5}\right)}\left(s^{2}+A s+B\right)}{s^{2}+\frac{2 K_{7}}{C_{1}}\left(V_{C 4}-V_{C S}\right) s+\frac{4 K_{3} K_{7} K_{5}\left(V_{C D}-V_{C S}\right)\left(V_{C 2}-V_{C 1}\right)\left(V_{C 4}-V_{C S}\right)}{K_{8} C_{1} C_{2}\left(V_{C S}-V_{C 5}\right)}} \\
& A=\frac{2 K_{9}}{C_{1}}\left(V_{C 4}-V_{G}\right)+\frac{2 K_{1} K_{3}\left(V_{C Q}-V_{Q 0}\right)\left(V_{C 2}-V_{Q}\right)}{K_{2} C_{1}\left(V_{C 7}-V_{C Q}\right)} \\
& B=\frac{4 K_{9} K_{5} K_{6}\left(V_{C Q}-V_{C 0}\right)\left(V_{C 4}-V_{C B}\right)\left(V_{C 6}-V_{C S}\right)}{K_{2} C_{1} C_{2}\left(V_{C 7}-V_{C S}\right)}
\end{aligned}
$$

The $K_{i}$ coefficients, with $i=1 \ldots .6$, are relevant to the corresponding $\mathrm{MRCi}$, while $K_{7}$ is relevant to the two matched MOS transistors with gate voltages VC3 and VC4 and K8 is relevant to the two matched MOS transistors with gate voltages $\mathrm{V}_{\mathrm{C} 5}$ and $\mathrm{V}_{\mathrm{C} 6}$. In order to obtain stability, $\left(\mathrm{V}_{\mathrm{C} 4}-\mathrm{V}_{\mathrm{C} 3}\right)$ and $\left[\left(\mathrm{V}_{\mathrm{C} 10^{-}}-\mathrm{V}_{\mathrm{C} 9}\right)\left(\mathrm{V}_{\mathrm{C} 14^{-}}-\mathrm{V}_{\mathrm{C} 13}\right)\left(\mathrm{V}_{\mathrm{C} 12^{-}}\right.\right.$ $\left.\left.\mathrm{V}_{\mathrm{C} 11}\right)\right] /\left(\mathrm{V}_{\mathrm{C} 5}-\mathrm{V}_{\mathrm{C} 6}\right)$ must be positive. The transfer function coefficients can be orthogonally adjusted by acting on the gate voltages. To this end, if depletion transistors are used, it is possible to give a zero value to the gate voltages, because this kind of MOS can accept positive, zero or negative gate voltages. On the other hand, if enhancement transistors are used, the DC level of the signals has to be shifted for proper operation [5]. Furthermore, by suitably choosing the gate voltage differences and $K_{i}$ values, all the kinds of transfer functions can be obtained, both inverting and noninverting. For example:

- for the low-pass transfer function the coefficient of $\mathrm{s}^{2}$ is set to zero when $\mathrm{V}_{\mathrm{C} 7}-\mathrm{V}_{\mathrm{C} 8}=0$ and, consequently, the coefficient $\mathrm{A}$ is set to zero when $\mathrm{V}_{\mathrm{C} 2}-\mathrm{V}_{\mathrm{C} 1}=0$;

- for the band-pass transfer function the coefficient of $\mathrm{s}^{2}$ is set to zero when $\mathrm{V}_{\mathrm{C} 7}-\mathrm{V}_{\mathrm{C} 8}=0$ and the coefficient $\mathrm{B}$ is set to zero when $\mathrm{V}_{\mathrm{C} 16}-\mathrm{V}_{\mathrm{C} 15}=0$;

for the high-pass transfer function the coefficient $\mathrm{B}$ is set to zero when $\mathrm{V}_{\mathrm{C} 16}-\mathrm{V}_{\mathrm{C} 15}=0$ and the coefficient $\mathrm{A}$ is set to zero by suitably acting on $\mathrm{V}_{\mathrm{C} 2}-\mathrm{V}_{\mathrm{C} 1}$.

Finally for the presented circuit, considering that the terms $2 \mathrm{~K}_{\mathrm{i}}\left(\mathrm{V}_{\mathrm{Cj}}-\mathrm{V}_{\mathrm{Cr}}\right)$ represent conductances [2, 4], 
the sensitivities of the transfer function coefficients, with respect to the components, are within the range $0 \leq|\mathrm{S}| \leq 1$ and, thus, are quite low.

\section{Simulation results}

In order to verify the obtained theoretical results, the circuit in Fig. 2 has been simulated with PSPICE. The $\mathrm{K}$ value depends on the physical characteristics of circuit in Fig. 2 is shown. The curves are referred to two different values of $\omega p$ obtained by varying $\mathrm{V}_{\mathrm{C} 12}-\mathrm{V}_{\mathrm{C} 11}$ :

$$
\mathrm{V}_{\mathrm{C} 12}-\mathrm{V}_{\mathrm{C} 11}=1 \mathrm{~V}\left(\omega \mathrm{p}=10^{5} \mathrm{rad} / \mathrm{sec}, \mathrm{Qp}=5\right)
$$

for the curve marked $\diamond$

$$
\mathrm{V}_{\mathrm{C} 12}-\mathrm{V}_{\mathrm{C} 11}=4 \mathrm{~V} \quad\left(\omega \mathrm{p}=2 \cdot 10^{5} \mathrm{rad} / \mathrm{sec}, \mathrm{Qp}=10\right)
$$
for the curve marked $\Delta$.

The other gate voltage differences are the following:

$\mathrm{V}_{\mathrm{C} 7}-\mathrm{V}_{\mathrm{C} 8}=\mathrm{V}_{\mathrm{C} 2}-\mathrm{V}_{\mathrm{C} 1}=0$

$\mathrm{V}_{\mathrm{C} 5}-\mathrm{V}_{\mathrm{C} 6}=\mathrm{V}_{\mathrm{C} 10}-\mathrm{V}_{\mathrm{C} 9}=\mathrm{V}_{\mathrm{C} 14}-\mathrm{V}_{\mathrm{C} 1} 3=1 \mathrm{~V}$

$\mathrm{V}_{\mathrm{C} 4}-\mathrm{V}_{\mathrm{C} 3}=0.2 \mathrm{~V} \quad \mathrm{~V}_{\mathrm{C} 15}-\mathrm{V}_{\mathrm{C} 16}=1 \mathrm{~V}$.

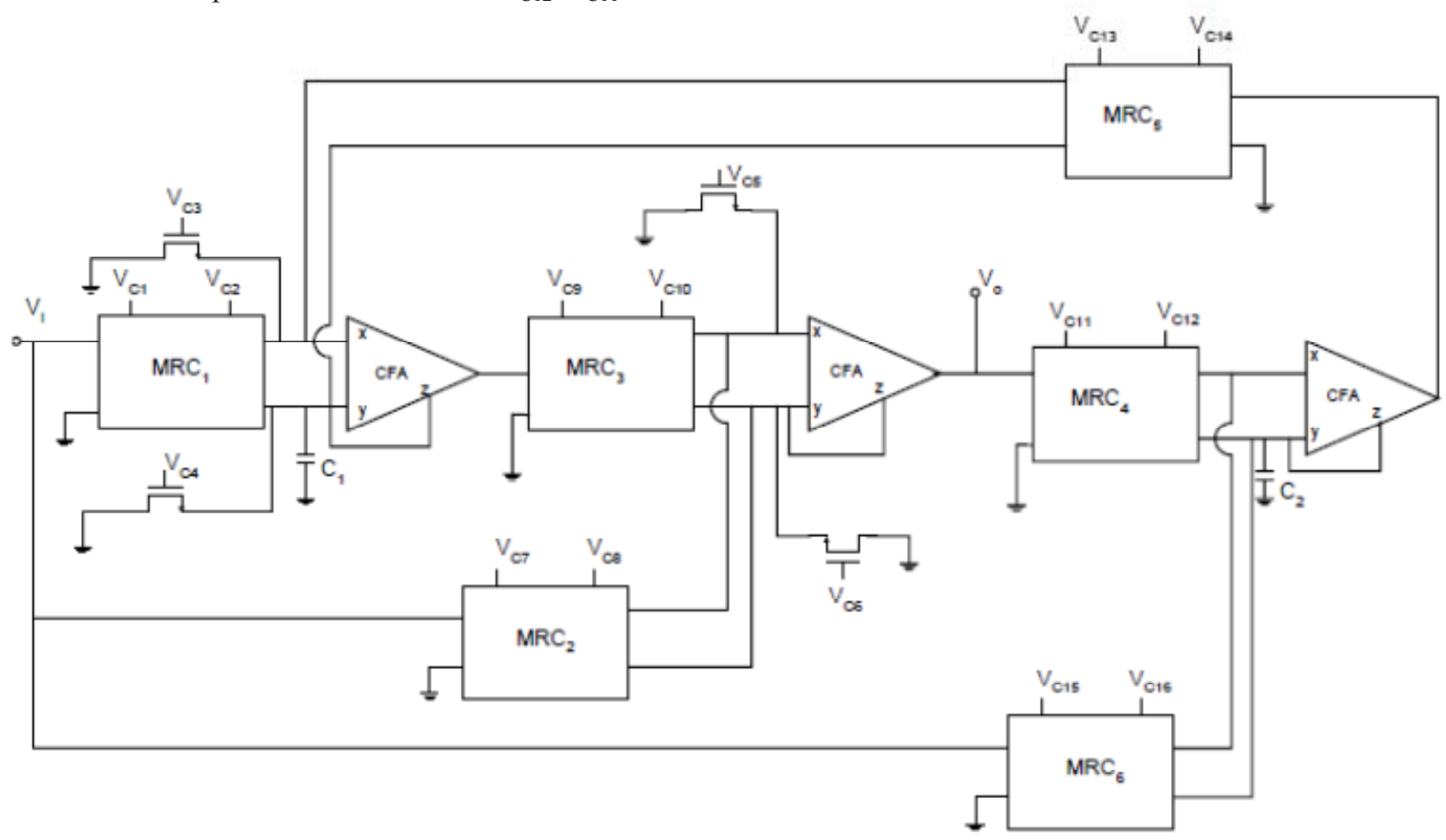

Fig. 2. Universal biquad with CFAs

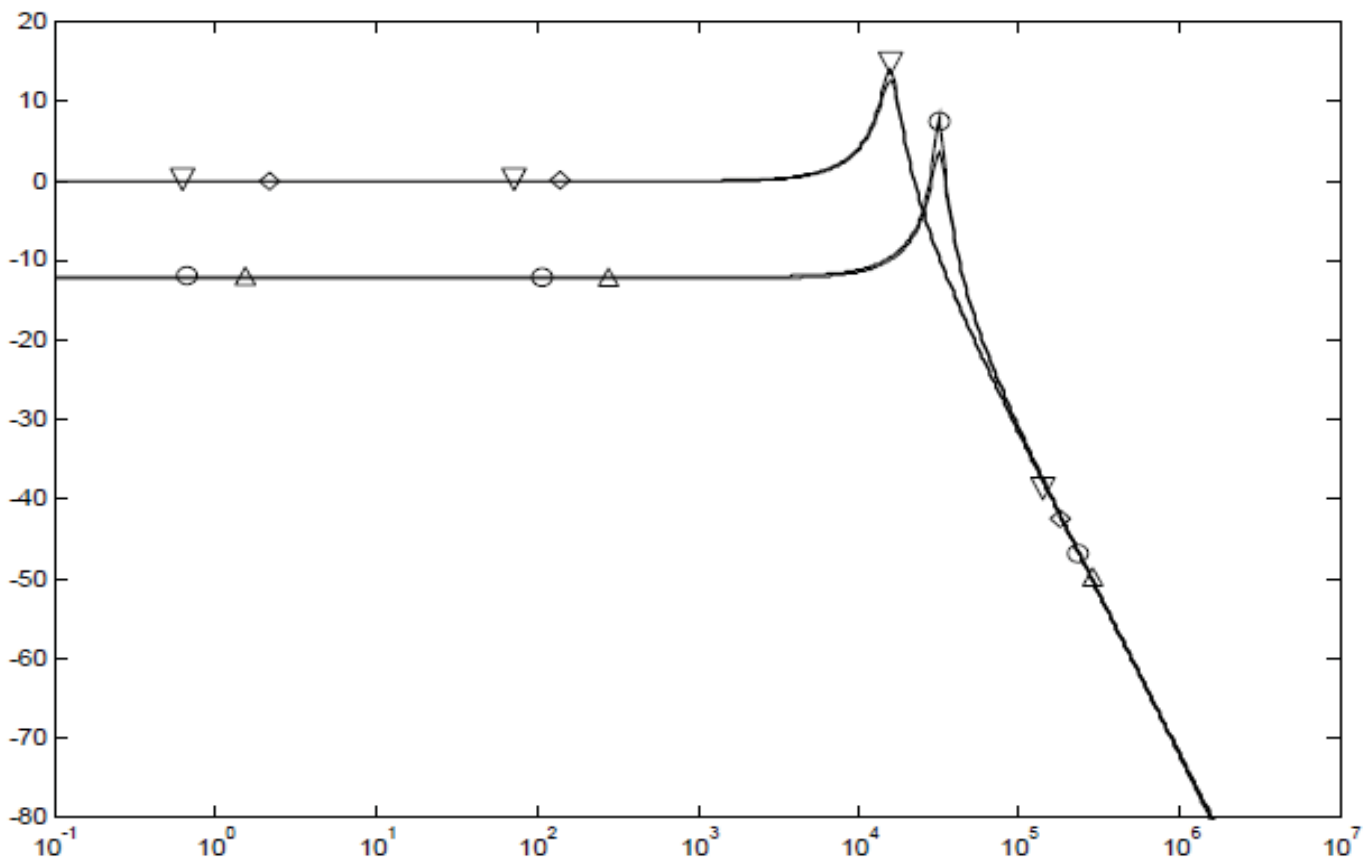

Fig. 3. Gain for a low-pass response of the circuit in Fig. 2: $\diamond \nabla \mathrm{V}_{\mathrm{C} 12}-\mathrm{V}_{\mathrm{C} 11}=1 \mathrm{~V}$, $\Delta \mathrm{O} \mathrm{V}_{\mathrm{C} 12}-\mathrm{V}_{\mathrm{C} 11}=4 \mathrm{~V}(\diamond \Delta \mathrm{AD} 844$ SPICE model $\nabla$ O CFA ideal model $)$ 


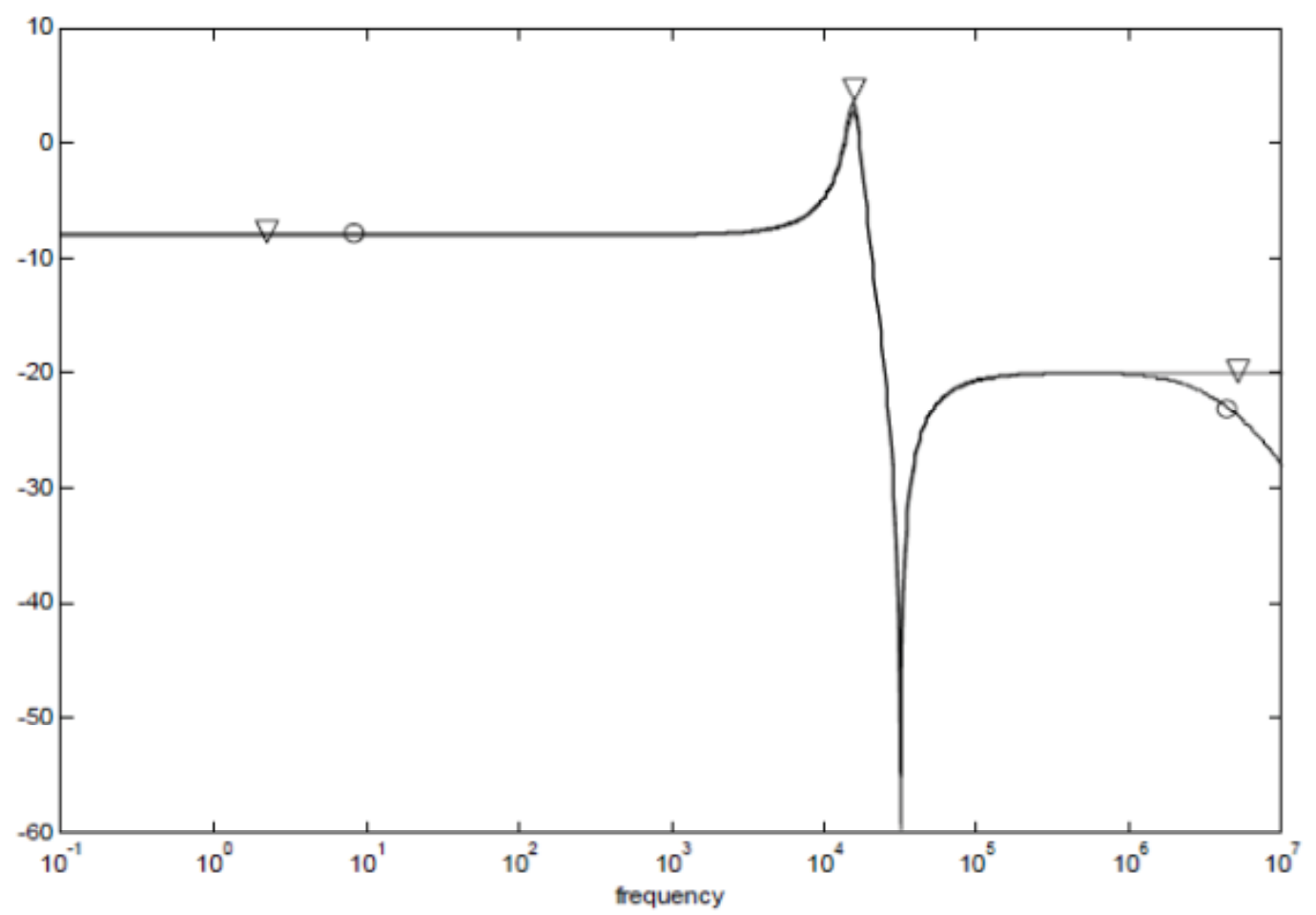

Fig. 4. Gain for a low-pass notch response of the circuit in Fig. 2 (O AD844 SPICE model $\nabla$ CFA ideal model)

In Fig. 4 the gain $(\mathrm{dB})$ for a low-pass notch response is shown. The gate voltage differences are the following:

$$
\begin{array}{lr}
\mathrm{V}_{\mathrm{C} 5}-\mathrm{V}_{\mathrm{C} 6}=\mathrm{V}_{\mathrm{C} 10}-\mathrm{V}_{\mathrm{C} 9}=\mathrm{V}_{\mathrm{C} 12}-\mathrm{V}_{\mathrm{C} 11}=\mathrm{V}_{\mathrm{C} 14}-\mathrm{V}_{\mathrm{C} 13}=1 \mathrm{~V} \\
\mathrm{~V}_{\mathrm{C} 2}-\mathrm{V}_{\mathrm{Cl}}=0.02 \mathrm{~V} & \mathrm{~V}_{\mathrm{C} 4}-\mathrm{V}_{\mathrm{C} 3}=0.2 \mathrm{~V} \\
\mathrm{~V}_{\mathrm{C} 15}-\mathrm{V}_{\mathrm{C} 16}=0.4 \mathrm{~V} & \mathrm{~V}_{\mathrm{C} 7}-\mathrm{V}_{\mathrm{C} 8}=0.1 \mathrm{~V}
\end{array}
$$

As a consequence $\omega_{\mathrm{p}}=105 \mathrm{rad} / \mathrm{sec}, \omega_{\mathrm{z}}=2 \cdot 105 \mathrm{rad} / \mathrm{sec}$ and $\mathrm{Q}_{\mathrm{p}}=5$.

The simulated frequency responses agree with theory.

\section{Conclusions}

A universal biquad using current feedback amplifiers as active devices and a canonical number of capacitors has been presented. It realizes the classical feedforward structure through the use of grounded capacitors and MOS transistors, which permit a fully integrability. The circuit offers the following advantages: electronic control of the transfer function coefficients, cascadability, low sensitivity, only grounded capacitors. Finally the simulation results confirm the theoretical analysis.

\section{References}

[1] S. Franco, "Design with operational amplifiers and analog integrated circuits", McGraw-Hill, New York, 1998.

[2] M. C. Piccirilli, "A fully integrated Tow-Thomas biquad using current feedback amplifiers", Proc. International Conference on Electronics, Circuits and Systems, Pafos, Cyprus, pp.1325-1328, 1999.

[3] G. Daryanani, "Principles of active network synthesis and design", John Wiley, New York, 1976.

[4] S. Takagi, Z. Czarnul, T. Iida, N. Fujii, "Generalization of MRC circuits and its applications", IEEE Trans. on Circuits and Systems-I, Vol. 44, No. 9, pp. 777-784, 1997.

[5] N. I. Khachab, M. Ismail, "MOS multiplier/divider cell for analogue VLSI", Electronics Letters, Vol. 25, No. 23, pp. 1550-1552, 1989. 